\title{
Comparing the Approval and Coverage Decisions of New Oncology Drugs in the United States and Other Selected Countries
}

\author{
Yuting Zhang, PhD; Hana Chantel Hueser; and Inmaculada Hernandez, PharmD, PhD
}

\begin{abstract}
BACKGROUND: Global pharmaceutical sales for anticancer drugs were $\$ 74.4$ billion in 2014, ranking first for drugs by therapeutic class. Countries may differ substantially in the approval and coverage decisions for anticancer drugs. OBJECTIVE: To compare the approval and coverage decisions for new anticancer drugs between the United States and 4 other countries: the United Kingdom, France, Australia, and Canada.

METHODS: We identified all new anticancer drug indications approved by the FDA between January 1, 2009, and December 31, 2013. For each country, we reviewed the organizations, processes, criteria, and special considerations used to make approval and coverage decisions for the drug indications approved. We further quantified and compared the variations across the 5 countries in the approval and coverage decisions as of June 30,2014 , for new anticancer drug indications.

RESULTS: Of 45 anticancer drug indications approved in the United States between January 1, 2009, and December 31, 2013, 67\% (30) were approved by the European Medicines Agency, and 53\% (24) were approved in Canada and Australia before December 31, 2013. The U.S. Medicare program covered all 45 drug indications, and as of June 30,2014 , the United Kingdom covered $87 \%(26)$ of those approved in Europe-58\% (26) of the drug indications covered by Medicare. France, Canada, and Australia covered $42 \%(19), 29 \%$ (13), and $24 \%$ (11) of the drug indications covered by Medicare, respectively.
\end{abstract}

CONCLUSIONS: Approval and reimbursement decisions vary substantially by country. The United States had the fewest access restrictions, and Australia was the most restrictive of the 5 countries that were examined.

J Manag Care Spec Pharm. 2017;23(2):247-54

Copyright $\odot 2017$, Academy of Managed Care Pharmacy. All rights reserved.

\section{What is already known about this subject}

Previous studies have shown that $42 \%$ of anticancer drugs approved in the United States from 2004 to 2008 were approved in the United Kingdom, and 35\% of anticancer drugs approved in the United States from 2000 to 2009 were approved in Australia. The U.S. Medicare program covers anticancer drugs through its Part B and Part D components: Oncologic drugs that need to be administrated by physicians are generally covered by Part B with a fixed $20 \%$ coinsurance, and oral anticancer drugs are covered under Medicare Part D with varying coinsurance depending on plan formularies and benefit structures.

Australia, Canada, and the United Kingdom use cost-effectiveness analyses explicitly, and France considers costs implicitly in its coverage decisions.

\section{What this study adds}

This study updates the previous country comparisons to cover the years 2009-2013 and expands the comparisons to France and Canada.

Among the 5 countries reviewed, the United States had the fewest access restrictions, and Australia had the most restrictions.

Countries using cost-effectiveness analyses explicitly are more restrictive in their coverage decisions.

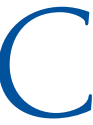

ancer is among the leading causes of morbidity and mortality worldwide. In 2012 alone, there were 14 million new cancer cases worldwide, 8.2 million cancerrelated deaths, and 32.6 million people living with cancer. ${ }^{1}$ Cancer treatment is costly. Anticancer drugs are estimated to account for $12 \%$ of total direct cancer care costs and $5 \%$ of total drug costs worldwide. ${ }^{2}$ In 2014, global pharmaceutical sales for anticancer drugs were $\$ 74.4$ billion, ranking first in global sales for drugs by therapeutic class. ${ }^{3}$

Scientific understanding of cancer is growing rapidly and has led to a surge in new development of anticancer drugs. Approval and reimbursement decisions may vary substantially by country. Many national health care systems use strategies to rationally allocate scarce health care resources. ${ }^{4}$ For instance, the United Kingdom bases its coverage decisions on health technology assessments that include a cost-effectiveness analysis, that is, a drug is covered if it is below a prespecified cost per quality-adjusted life-year (QALY). However, restricting access to anticancer drugs is highly controversial because of the lack of therapeutic alternatives for some types of cancer.

In this study, we compared the approval and coverage decisions for new anticancer drugs between the United States and 4 other countries: the United Kingdom, France, Australia, and Canada. These 4 countries were chosen because, compared with the United States, they accomplish better health outcomes-commonly measured by life expectancy, infant mortality, and percentage of population with multiple chronic conditions - with less than or about half of the total health care expenditures per capita in the United States. ${ }^{5}$ All 5 countries rely on a market economy and share similar political systems. France, the United Kingdom, and Australia often rank at the top based on measures such as efficiency and equity, but each has its unique way for allocating health care resources. ${ }^{5,6}$ 
Canada was also chosen for its geographic proximity to the United States. We reviewed the organizations, processes, criteria, and special considerations used by these countries to make approval and coverage decisions for new anticancer drug indications that were approved by the U.S. Food and Drug Administration (FDA) between January 1, 2009, and December 31, 2013. We further quantified and compared the variations across the 5 countries of the approval and coverage decisions for these new anticancer drug indications before June 30, 2014.

\section{An Overview of the International Comparison of Anticancer Drug Approval and Coverage}

United States. In the United States, the FDA's Center for Drug Evaluation and Research evaluates the safety and efficacy of new medications and makes decisions about whether a new drug should enter the U.S. market on the basis of safety, quality, and efficacy. ${ }^{7}$ Cost is not a criterion used for approving a new drug. For anticancer drugs, the FDA Office of Hematology and Oncology Drug Products facilitates the rapid review of promising new cancer therapies.

Once a new drug is approved by the FDA, public and private insurance programs separately evaluate the coverage decisions for their own covered patients. There are 3 main publicly funded insurance programs: Medicare covers the elderly and disabled; Medicaid covers the poor; and the Department of Veterans Affairs covers veterans. Medicare covers anticancer drugs through its Part B and Part D programs. Oncologic drugs that need to be administrated by physicians are generally covered by Part B, with a fixed $20 \%$ coinsurance after a deductible, and the law requires Medicare Part B to cover any drug used in an "anticancer chemotherapeutic regimen," as long as the use is "for a medically accepted indication," which includes off-label use-an indication not approved by the FDA but accepted by physicians as medically beneficial treatment. ${ }^{8}$ Oral anticancer drugs, which patients can obtain from a pharmacy, are covered under Medicare Part $\mathrm{D}$, with varying coinsurance depending on plan formularies and benefit structures. For veterans, anticancer drugs are covered, as long as the drug is used for an approved indication. For individuals with commercial insurance covering prescription drugs, each insurance plan maintains a formulary, which is a list that indicates what prescription drugs are covered by the plan and the rate of copayments or coinsurance for the drugs.

United Kingdom. During the study period, the United Kingdom was a member of the European Union, where the European Medicines Agency (EMA) appraises the safety and efficacy of new medications and supervises their entrance to and duration on the market in the European Union. The EMA applies its centralized procedure to determine which medications are approved to enter the market in all European Union member countries.
For the United Kingdom specifically, the National Institute for Clinical Excellence (NICE) assesses the technologies referred by the U.K. Department of Health and issues coverage recommendations to the U.K. National Health Service (NHS). ${ }^{11}$ NICE recommendations are based on drug efficacy, safety, and cost-effectiveness. NICE cost-effectiveness coverage threshold for cancer drugs is $£ 50,000$ (U.S. \$74,500) per QALY, which is $£ 12,000$ (U.S. $\$ 17,880$ ) per QALY higher than for the rest of the drug classes set at $£ 38,000$ (U.S. $\$ 56,620$ ) per QALY. ${ }^{12}$ In addition, the Cancer Drugs Fund provides $£ 200$ million (U.S. \$298 million) each year to cover anticancer drugs not covered by the NHS, and some pharmaceutical companies have negotiated patient access schemes with the NHS for drugs not recommended by NICE. ${ }^{13}$ These risk-sharing contracts allow patients to have access to some drugs not covered otherwise.$^{14}$ Almost half of the drugs with currently approved patient access schemes are anticancer drugs. ${ }^{15}$

France. After a drug is approved by the EMA to enter the European market, the French Agency for the Safety of Medicines and Health Products must approve all medications for use in France. ${ }^{16}$

The Transparency Committee of the High Authority of Health recommends the coverage of new drugs on the market in France. The committee appraises the severity of the drug's cancer indication; its effectiveness; its safety profile; whether it is intended to prevent, cure, or relieve symptoms; and how it compares to other drugs on the market. ${ }^{17}$ The National Union of Health Insurance Funds uses the determinations of the Transparency Committee, as well as the severity of the indication, to define the rate of permissible reimbursement benefits and fixed rate support care for the drug. Orphan drugs seen as irreplaceable and lifesaving receive 100\% reimbursement, and this applies to many of the available cancer drugs on the List of Long-Term Afflictions. ${ }^{18}$

Australia. The Australian Therapeutic Goods Administration approves new drugs to enter the Australian market. The approval decision is based on the efficacy, cost-effectiveness, and safety of a drug. ${ }^{19,20}$

The Pharmaceutical Benefits Advisory Committee is responsible for the coverage decisions in Australia after a drug is approved ${ }^{21} \mathrm{~A}$ coverage decision is based on the efficacy, costeffectiveness, and safety of the drug as compared with other treatments currently covered for the same indication. There is no stated cost-effectiveness threshold, but the regularly implied level is AUD \$50,000 (U.S. \$39,000) per QALY.22 The only exception to this pathway for coverage is for orphan drugs, whose coverage is usually rejected by the Pharmaceutical Benefits Advisory Committee based on cost-effectiveness and is reconsidered under the Life Saving Drugs Program. Under this program, orphan drugs for rare and life-threatening forms of cancer are covered for eligible patients. 
Canada. Health Canada's drug review process begins with its Therapeutic Products Directorate, a board of scientists that assesses the quality, safety, and efficacy of a drug and decides whether the benefits outweigh the risks of allowing the drug to enter and continue to be on the market. ${ }^{23}$

The Canadian Agency for Drugs and Technologies in Health runs a Common Drug Review to rate the clinical and cost effectiveness of drugs for the purpose of recommending drugs to be covered by the provinces, but each province can make its own decision. ${ }^{24}$ The pan-Canadian Oncology Drug Review evaluates oncology drugs based on clinical evidence and costeffectiveness, recommends funding decisions, and includes suggested dosage and place in therapy..$^{25}$ Then, the provinces make their respective coverage decisions independently on the basis of the information provided by the pan-Canadian Oncology Drug Review.

\section{Methods}

\section{Identification of FDA-Approved New Oncology Drugs}

We identified new drugs approved by the FDA for the treatment of any cancer between January 1, 2009, and December 31, 2013, using the FDA (http://www.fda.gov/) and CenterWatch (http://www.centerwatch.com/) websites (Table 1). Previous researchers have shown that new drugs are approved more quickly in the United States, compared with European countries, so using the list of new drugs approved by the FDA provided the most updated list of new drugs. ${ }^{26,27}$ Our sample included active ingredients approved for new cancer indications between January 1, 2009, and December 31, 2013. Pharmaceutical agents used to treat any chemotherapy-induced side effects or cancer-related pains were excluded. The final list included 41 unique drugs that were approved for 45 drug indications related to cancer. We also stratified the analyses by each drug's route of administration: oral or injectable.

\section{Identification of Approval Data for New Anticancer Drugs}

The following websites were checked to obtain approval dates between January 1, 2009, and December 31, 2013: EMA (http://www.ema.europa.eu/ema/) for the United Kingdom and France; the French Agency for the Safety of Medicines and Health Products for France (http://agence-prd.ansm.sante. fr/php/ecodex/index.php); the Australian Therapeutic Goods Administration (http://www.tga.gov.au/) for Australia; and the Drug Product Database on the Health Canada website (http:// webprod5.hc-sc.gc.ca/dpd-bdpp/index-eng.jsp) for Canada.

\section{Identification of Coverage Decision Data of New Anticancer Drugs}

Between March and June 2014, we checked the following websites to collect coverage information for all approved drug indications in each country. We reviewed the British National Formulary websites to identify whether approved drugs were covered by the NHS. ${ }^{28}$ For drug coverage in France, we reviewed the Public Database of Medications and the Technical Agency for Hospital Information (Agence Technique de l'Information sur l'Hospitalisation). ${ }^{29,30}$ We searched the Pharmaceutical Benefits Advisory Committee's website to determine whether a drug was covered in Australia. ${ }^{31}$ The pan-Canadian Oncology Drug Review database was used to identify coverage data for the drugs approved in Canada. ${ }^{32}$ Because all the study drugs were approved and covered by Medicare in the United States, we used Medicare as a benchmark to compare with the other countries. We calculated the percentages of approved U.S. drugs that were approved, approved and covered, and approved but not covered in other countries. If a drug was approved before December 31, 2013, in other countries, it was defined as approved; if a drug was covered at the time of collecting these data, it was defined as covered.

\section{Results}

Before December 31, 2013, 67\% (30) of those 45 drug indications were approved by the EMA and therefore available (but possibly not covered) in the United Kingdom, and France approved all 30 EMA-approved drug indications. In Canada and Australia, 53\% (24) of the drug indications were approved.

All these drug indications were covered by Medicare, with 23 covered in Medicare Part B and 22 covered in Medicare Part D. Figure 1 summarizes the percentage of drugs covered by Medicare that were approved and covered by other countries as of June 30,2014, showing the total as well as stratifying by route of administration. The NHS covered $87 \%$ (26) of the 30 drugs approved in the United Kingdom, or 58\% (26) of the 45 drug indications covered by Medicare. France covered 63\% of the 30 drug indications approved in France, equivalent to $42 \%$ (19) of what Medicare covered, followed by Canada with 29\% (13) and Australia with 24\% (11) of what Medicare covered.

After stratifying by route of administration, we found that the coverage of oral anticancer drugs is less restrictive than the coverage of injections in all non-U.S. countries. Specifically, the United Kingdom covered $71 \%$ (17) of all oral anticancer drug-indications in our list, but only $43 \%$ (9) of the injectable anticancer drug-indications"

\section{Discussion}

We compared the approval and coverage decisions in 5 developed countries for 45 new cancer drug indications that were approved by the FDA between January 1, 2009, and December 31, 2013. Medicare covered all 45 drug indications approved. The list of 5 countries in order from the most restrictive to the least restrictive is Australia, Canada, France, the United Kingdom, and the United States.

Mason et al. (2010) compared the coverage decisions in the United States and the United Kingdom for 46 anticancer drugs 
TABLE 1 List of New Oncology Drugs Approved by the FDA, January 1, 2009-December 31, 2013

\begin{tabular}{|c|c|c|c|c|c|c|c|c|c|c|c|}
\hline \multirow[b]{2}{*}{$\begin{array}{l}\text { Brand } \\
\text { Name }\end{array}$} & \multirow[b]{2}{*}{$\begin{array}{c}\text { Active } \\
\text { Ingredient }\end{array}$} & \multirow[b]{2}{*}{ Indication } & \multirow[b]{2}{*}{$\begin{array}{c}\text { Route of } \\
\text { Administration }\end{array}$} & \multicolumn{4}{|c|}{ Approval Dates } & \multicolumn{4}{|c|}{ Coverage } \\
\hline & & & & FDA & EMA & Canada & Australia & $\begin{array}{c}\text { United } \\
\text { Kingdom }\end{array}$ & France & Canada & Australia \\
\hline Abraxane & $\begin{array}{c}\text { Paclitaxel } \\
\text { protein-bound } \\
\text { particles }\end{array}$ & $\begin{array}{l}\text { Non-small cell lung } \\
\text { cancer }\end{array}$ & Injectable & $\begin{array}{l}\text { October } \\
2012\end{array}$ & $\begin{array}{c}\text { Not } \\
\text { Approved }\end{array}$ & $\begin{array}{c}\text { Not } \\
\text { Approved }\end{array}$ & \begin{tabular}{|c|} 
Not \\
Approved
\end{tabular} & NA & NA & NA & NA \\
\hline Adcetris & $\begin{array}{l}\text { Brentuximab } \\
\text { vedotin }\end{array}$ & $\begin{array}{l}\text { Hodgkin lymphoma } \\
\text { and anaplastic large } \\
\text { cell lymphoma }\end{array}$ & Injectable & $\begin{array}{l}\text { August } \\
2011\end{array}$ & $\begin{array}{l}\text { October } \\
2012\end{array}$ & \begin{tabular}{|c|} 
February \\
2013
\end{tabular} & \begin{tabular}{|c|} 
December \\
2013
\end{tabular} & Yes & Yes & Yes & No \\
\hline Afinitor & Everolimus & Renal cell carcinoma & Oral & $\begin{array}{l}\text { March } \\
2009\end{array}$ & $\begin{array}{c}\text { August } \\
2009\end{array}$ & $\begin{array}{c}\text { Not } \\
\text { Approved }\end{array}$ & $\begin{array}{c}\text { August } \\
2009\end{array}$ & Yes & Yes & NA & Yes \\
\hline Afinitor & Everolimus & $\begin{array}{l}\text { Advanced pancre- } \\
\text { atic neuroendocrine } \\
\text { tumors }\end{array}$ & Oral & $\begin{array}{l}\text { May } \\
2011\end{array}$ & $\begin{array}{c}\text { September } \\
2011\end{array}$ & $\begin{array}{c}\text { August } \\
2011\end{array}$ & $\begin{array}{l}\text { July } \\
2012\end{array}$ & Yes & Yes & Yes & Yes \\
\hline Afinitor & Everolimus & $\begin{array}{l}\text { Hormone receptor- } \\
\text { positive, HER2- } \\
\text { negative breast } \\
\text { cancer }\end{array}$ & Oral & $\begin{array}{l}\text { July } \\
2012\end{array}$ & $\begin{array}{l}\text { July } \\
2012\end{array}$ & $\begin{array}{l}\text { January } \\
2013\end{array}$ & $\begin{array}{c}\text { February } \\
2013\end{array}$ & Yes & Yes & Yes & Yes \\
\hline Arzerra & Ofatumumab & $\begin{array}{l}\text { Chronic lymphocytic } \\
\text { leukemia }\end{array}$ & Injectable & $\begin{array}{l}\text { October } \\
2009\end{array}$ & $\begin{array}{l}\text { April } \\
2010\end{array}$ & $\begin{array}{l}\text { August } \\
2012\end{array}$ & $\begin{array}{c}\text { Not } \\
\text { Approved }\end{array}$ & Yes & No & No & NA \\
\hline Avastin & Bevacizumab & Renal cell carcinoma & Injectable & $\begin{array}{l}\text { July } \\
2009\end{array}$ & $\begin{array}{c}\text { January } \\
2008\end{array}$ & $\begin{array}{c}\text { Not } \\
\text { Approved }\end{array}$ & $\begin{array}{c}\text { Not } \\
\text { Approved }\end{array}$ & Yes & No & NA & NA \\
\hline Bosulif & Bosutinib & $\begin{array}{l}\mathrm{Ph}+\text { chronic myelog- } \\
\text { enous leukemia }\end{array}$ & Oral & $\begin{array}{c}\text { September } \\
2012\end{array}$ & $\begin{array}{l}\text { March } \\
2013\end{array}$ & $\begin{array}{c}\text { Not } \\
\text { Approved }\end{array}$ & $\begin{array}{c}\text { Not } \\
\text { Approved }\end{array}$ & Yes & Yes & NA & NA \\
\hline Cometriq & Cabozantinib & $\begin{array}{l}\text { Metastatic medullary } \\
\text { thyroid cancer }\end{array}$ & Oral & $\begin{array}{c}\text { November } \\
2012\end{array}$ & $\begin{array}{c}\text { Not } \\
\text { Approved }\end{array}$ & $\begin{array}{c}\text { Not } \\
\text { Approved }\end{array}$ & $\begin{array}{c}\text { Not } \\
\text { Approved }\end{array}$ & NA & NA & $\mathrm{NA}$ & NA \\
\hline Erivedge & Vismodegib & Basal cell carcinoma & Oral & $\begin{array}{l}\text { January } \\
2012\end{array}$ & $\begin{array}{l}\text { July } \\
2013\end{array}$ & $\begin{array}{l}\text { August } \\
2013\end{array}$ & $\begin{array}{l}\text { May } \\
2013\end{array}$ & Yes & Yes & Yes & No \\
\hline Erwinaze & $\begin{array}{l}\text { Asparaginase } \\
\text { Erwinia } \\
\text { chrysanthemi }\end{array}$ & $\begin{array}{l}\text { Acute lymphoblastic } \\
\text { leukemia }\end{array}$ & Injectable & $\begin{array}{c}\text { November } \\
2011\end{array}$ & $\begin{array}{c}\text { Not } \\
\text { Approved }\end{array}$ & $\begin{array}{c}\text { Not } \\
\text { Approved }\end{array}$ & $\begin{array}{c}\text { Not } \\
\text { Approved }\end{array}$ & NA & NA & NA & NA \\
\hline Folotyn & Pralatrexate & $\begin{array}{l}\text { Peripheral T-cell } \\
\text { lymphoma }\end{array}$ & Injectable & $\begin{array}{c}\text { September } \\
2009\end{array}$ & \begin{tabular}{c|} 
Not \\
Approved
\end{tabular} & $\begin{array}{c}\text { Not } \\
\text { Approved }\end{array}$ & $\begin{array}{c}\text { Not } \\
\text { Approved }\end{array}$ & NA & NA & NA & NA \\
\hline Gazyva & Obinutuzumab & $\begin{array}{l}\text { Previously untreated } \\
\text { chronic lymphocytic } \\
\text { leukemia }\end{array}$ & Injectable & $\begin{array}{l}\text { October } \\
2013\end{array}$ & $\begin{array}{c}\text { Not } \\
\text { Approved }\end{array}$ & $\begin{array}{c}\text { Not } \\
\text { Approved }\end{array}$ & $\begin{array}{c}\text { Not } \\
\text { Approved }\end{array}$ & NA & NA & NA & NA \\
\hline Gilotrif & Afatinib & $\begin{array}{l}\text { Metastatic non-small } \\
\text { cell lung cancer with } \\
\text { EGFR mutations }\end{array}$ & Oral & $\begin{array}{l}\text { July } \\
2013\end{array}$ & $\begin{array}{c}\text { September } \\
2013\end{array}$ & $\begin{array}{c}\text { Not } \\
\text { Approved }\end{array}$ & $\begin{array}{c}\text { November } \\
2013\end{array}$ & No & Yes & NA & No \\
\hline Halaven & $\begin{array}{l}\text { Eribulin } \\
\text { mesylate }\end{array}$ & $\begin{array}{l}\text { Metastatic breast } \\
\text { cancer }\end{array}$ & Injectable & $\begin{array}{c}\text { November } \\
2010\end{array}$ & $\begin{array}{l}\text { March } \\
2011\end{array}$ & $\begin{array}{l}\text { March } \\
2012\end{array}$ & $\begin{array}{l}\text { August } \\
2012\end{array}$ & Yes & Yes & Yes & No \\
\hline Herceptin & Trastuzumab & Gastric cancer & Injectable & $\begin{array}{c}\text { October } \\
2010\end{array}$ & $\begin{array}{l}\text { January } \\
2010\end{array}$ & $\begin{array}{c}\text { August } \\
2010\end{array}$ & $\begin{array}{c}\text { September } \\
2010\end{array}$ & Yes & Yes & No & Yes \\
\hline Iclusig & Ponatinib & $\begin{array}{l}\text { Chronic myeloid } \\
\text { leukemia and } \\
\text { Philadelphia chromo- } \\
\text { some positive acute } \\
\text { lymphoblastic } \\
\text { leukemia }\end{array}$ & Oral & $\begin{array}{c}\text { December } \\
2012\end{array}$ & $\begin{array}{l}\text { July } \\
2013\end{array}$ & $\begin{array}{c}\text { Not } \\
\text { Approved }\end{array}$ & $\begin{array}{c}\text { Not } \\
\text { Approved }\end{array}$ & Yes & No & NA & NA \\
\hline Imbruvica & Ibrutinib & $\begin{array}{l}\text { Mantle cell } \\
\text { lymphoma }\end{array}$ & Oral & \begin{tabular}{|c|} 
November \\
2013
\end{tabular} & \begin{tabular}{|c|} 
Not \\
Approved
\end{tabular} & $\begin{array}{c}\text { Not } \\
\text { Approved }\end{array}$ & $\begin{array}{c}\text { Not } \\
\text { Approved }\end{array}$ & NA & NA & NA & NA \\
\hline Inlyta & Axitinib & $\begin{array}{l}\text { Advanced renal cell } \\
\text { carcinoma }\end{array}$ & Oral & $\begin{array}{l}\text { January } \\
2012\end{array}$ & $\begin{array}{c}\text { September } \\
2012\end{array}$ & $\begin{array}{c}\text { August } \\
2012\end{array}$ & $\begin{array}{l}\text { July } \\
2012\end{array}$ & Yes & Yes & Yes & No \\
\hline Istodax & Romidepsin & $\begin{array}{l}\text { Cutaneous T-cell } \\
\text { lymphoma }\end{array}$ & Injectable & $\begin{array}{c}\text { November } \\
2009\end{array}$ & \begin{tabular}{c|} 
Not \\
Approved
\end{tabular} & \begin{tabular}{|c|} 
Not \\
Approved
\end{tabular} & $\begin{array}{l}\text { August } \\
2013\end{array}$ & NA & NA & NA & No \\
\hline Jevtana & Cabazitaxel & Prostate cancer & Injectable & $\begin{array}{l}\text { June } \\
2010\end{array}$ & $\begin{array}{c}\text { March } \\
2011\end{array}$ & $\begin{array}{c}\text { August } \\
2011\end{array}$ & $\begin{array}{c}\text { December } \\
2011\end{array}$ & Yes & Yes & No & Yes \\
\hline Kadcyla & $\begin{array}{l}\text { Ado- } \\
\text { trastuzumab }\end{array}$ & $\begin{array}{l}\text { HER2-positive meta- } \\
\text { static breast cancer }\end{array}$ & Injectable & \begin{tabular}{|c|} 
February \\
2013
\end{tabular} & $\begin{array}{c}\text { November } \\
2013\end{array}$ & $\begin{array}{l}\text { October } \\
2013\end{array}$ & $\begin{array}{c}\text { September } \\
2013\end{array}$ & No & Yes & Yes & No \\
\hline
\end{tabular}


TABLE 1 List of New Oncology Drugs Approved by the FDA, January 1, 2009-December 31, 2013 (continued)

\begin{tabular}{|c|c|c|c|c|c|c|c|c|c|c|c|}
\hline \multirow[b]{2}{*}{$\begin{array}{l}\text { Brand } \\
\text { Name }\end{array}$} & \multirow[b]{2}{*}{$\begin{array}{c}\text { Active } \\
\text { Ingredient }\end{array}$} & \multirow[b]{2}{*}{ Indication } & \multirow[b]{2}{*}{$\begin{array}{c}\text { Route of } \\
\text { Administration }\end{array}$} & \multicolumn{4}{|c|}{ Approval Dates } & \multicolumn{4}{|c|}{ Coverage } \\
\hline & & & & FDA & EMA & Canada & Australia & \begin{tabular}{|c|} 
United \\
Kingdom
\end{tabular} & France & Canada & Australia \\
\hline Kyprolis & Carfilzomib & Multiple myeloma & Injectable & July 2012 & \begin{tabular}{c|} 
Not \\
Approved
\end{tabular} & $\begin{array}{c}\text { Not } \\
\text { Approved }\end{array}$ & \begin{tabular}{|c|} 
Not \\
Approved
\end{tabular} & NA & NA & NA & NA \\
\hline Marchqibo & Vincristine & $\begin{array}{l}\text { Ph-acute lymphoblas- } \\
\text { tic leukemia }\end{array}$ & Injectable & $\begin{array}{l}\text { August } \\
2012\end{array}$ & \begin{tabular}{c|} 
Not \\
Approved
\end{tabular} & \begin{tabular}{|c|} 
Not \\
Approved
\end{tabular} & $\begin{array}{c}\text { Not } \\
\text { Approved }\end{array}$ & NA & NA & NA & NA \\
\hline Mekinist & Trametinib & $\begin{array}{l}\text { Unresectable or } \\
\text { metastatic melanoma } \\
\text { with BRAF V600E or } \\
\text { V600K mutations } \\
\end{array}$ & Oral & $\begin{array}{l}\text { May } \\
2013\end{array}$ & $\begin{array}{c}\text { Not } \\
\text { Approved }\end{array}$ & $\begin{array}{l}\text { August } \\
2013\end{array}$ & $\begin{array}{c}\text { Not } \\
\text { Approved }\end{array}$ & NA & NA & No & NA \\
\hline Perjeta & Pertuzumab & $\begin{array}{l}\text { HER2+ metastatic } \\
\text { breast cancer }\end{array}$ & Injectable & $\begin{array}{l}\text { June } \\
2012\end{array}$ & $\begin{array}{l}\text { March } \\
2013\end{array}$ & $\begin{array}{l}\text { May } \\
2013\end{array}$ & $\begin{array}{l}\text { May } \\
2013\end{array}$ & Yes & Yes & Yes & No \\
\hline Pomalyst & Pomalidomide & $\begin{array}{l}\text { Relapsed and } \\
\text { refractory multiple } \\
\text { myeloma }\end{array}$ & Oral & \begin{tabular}{|c|} 
February \\
2013
\end{tabular} & $\begin{array}{l}\text { August } \\
2013\end{array}$ & $\begin{array}{c}\text { Not } \\
\text { Approved }\end{array}$ & $\begin{array}{c}\text { Not } \\
\text { Approved }\end{array}$ & Yes & No & NA & NA \\
\hline Provenge & Sipuleucel-T & $\begin{array}{l}\text { Hormone refractory } \\
\text { prostate cancer }\end{array}$ & Injectable & $\begin{array}{l}\text { May } \\
2010\end{array}$ & $\begin{array}{c}\text { September } \\
2013\end{array}$ & $\begin{array}{c}\text { Not } \\
\text { Approved }\end{array}$ & \begin{tabular}{|c|} 
Not \\
Approved \\
\end{tabular} & No & No & NA & NA \\
\hline Revlimid & Lenalidomide & $\begin{array}{l}\text { Mantle cell } \\
\text { lymphoma }\end{array}$ & Oral & $\begin{array}{l}\text { June } \\
2013\end{array}$ & $\begin{array}{c}\text { Not } \\
\text { Approved }\end{array}$ & $\begin{array}{c}\text { Not } \\
\text { Approved }\end{array}$ & \begin{tabular}{|c|} 
Not \\
Approved \\
\end{tabular} & NA & NA & NA & NA \\
\hline Stivarga & Regorafenib & $\begin{array}{l}\text { Metastatic colorectal } \\
\text { cancer }\end{array}$ & Oral & \begin{tabular}{|c|} 
September \\
2012 \\
\end{tabular} & \begin{tabular}{|c|} 
September \\
2013
\end{tabular} & $\begin{array}{l}\text { April } \\
2013\end{array}$ & \begin{tabular}{|c|} 
November \\
2013
\end{tabular} & Yes & No & No & No \\
\hline Stivarga & Regorafenib & $\begin{array}{l}\text { Gastrointestinal } \\
\text { stromal tumor }\end{array}$ & Oral & \begin{tabular}{|c|} 
February \\
2013
\end{tabular} & \begin{tabular}{|c|} 
Not \\
Approved
\end{tabular} & $\begin{array}{l}\text { April } \\
2013\end{array}$ & $\begin{array}{c}\text { Not } \\
\text { Approved } \\
\end{array}$ & NA & NA & No & NA \\
\hline Sutent & Sunitinib & $\begin{array}{l}\text { Pancreatic neuroen- } \\
\text { docrine tumors }\end{array}$ & Oral & \begin{tabular}{|l|} 
May \\
2011 \\
\end{tabular} & $\begin{array}{c}\text { December } \\
2010\end{array}$ & $\begin{array}{c}\text { Not } \\
\text { Approved }\end{array}$ & $\begin{array}{c}\text { March } \\
2011\end{array}$ & Yes & Yes & NA & Yes \\
\hline Sylatron & $\begin{array}{c}\text { Peginterferon } \\
\text { alfa- } 2 \mathrm{~b}\end{array}$ & Melanoma & Injectable & $\begin{array}{l}\text { April } \\
2011\end{array}$ & $\begin{array}{c}\text { Not } \\
\text { Approved }\end{array}$ & \begin{tabular}{|c|} 
Not \\
Approved
\end{tabular} & \begin{tabular}{|c|} 
Not \\
Approved
\end{tabular} & NA & NA & NA & NA \\
\hline Synribo & Omacetaxine & $\begin{array}{l}\text { Chronic or acceler- } \\
\text { ated phase chronic } \\
\text { myeloid leukemia }\end{array}$ & Injectable & $\begin{array}{l}\text { October } \\
2012\end{array}$ & $\begin{array}{c}\text { Not } \\
\text { Approved }\end{array}$ & $\begin{array}{c}\text { Not } \\
\text { Approved }\end{array}$ & $\begin{array}{c}\text { Not } \\
\text { Approved }\end{array}$ & NA & NA & NA & NA \\
\hline Tafinlar & Dabrafenib & $\begin{array}{l}\text { Unresectable or } \\
\text { metastatic melanoma } \\
\text { with BRAF V600E } \\
\text { mutation }\end{array}$ & Oral & $\begin{array}{l}\text { May } \\
2013\end{array}$ & $\begin{array}{c}\text { September } \\
2013\end{array}$ & $\begin{array}{l}\text { August } \\
2013\end{array}$ & $\begin{array}{l}\text { August } \\
2013\end{array}$ & No & No & No & Yes \\
\hline Vandetanib & Vandetanib & Thyroid cancer & Oral & $\begin{array}{l}\text { April } \\
2011 \\
\end{array}$ & \begin{tabular}{|c|} 
February \\
2012 \\
\end{tabular} & \begin{tabular}{|c|} 
February \\
2012
\end{tabular} & $\begin{array}{c}\text { January } \\
2013 \\
\end{array}$ & Yes & Yes & No & No \\
\hline Votrient & Pazopanib & Renal cell carcinoma & Oral & $\begin{array}{c}\text { October } \\
2009\end{array}$ & $\begin{array}{l}\text { June } \\
2010\end{array}$ & $\begin{array}{l}\text { August } \\
2010\end{array}$ & $\begin{array}{l}\text { June } \\
2010\end{array}$ & Yes & No & Yes & Yes \\
\hline Votrient & Pazopanib & Soft tissue sarcoma & Oral & $\begin{array}{l}\text { April } \\
2012\end{array}$ & $\begin{array}{l}\text { August } \\
2012\end{array}$ & $\begin{array}{l}\text { August } \\
2010\end{array}$ & $\begin{array}{l}\text { May } \\
2011\end{array}$ & Yes & No & No & Yes \\
\hline Xalkori & Crizotinib & $\begin{array}{l}\text { ALK+ non-small cell } \\
\text { lung cancer }\end{array}$ & Oral & $\begin{array}{l}\text { August } \\
2011\end{array}$ & $\begin{array}{l}\text { October } \\
2012 \\
\end{array}$ & $\begin{array}{l}\text { May } \\
2012\end{array}$ & \begin{tabular}{|c|} 
September \\
2013 \\
\end{tabular} & Yes & Yes & No & No \\
\hline Xgeva & Denosumab & $\begin{array}{l}\text { Giant cell tumor of } \\
\text { bone }\end{array}$ & Injectable & $\begin{array}{l}\text { June } \\
2013 \\
\end{array}$ & $\begin{array}{c}\text { Not } \\
\text { Approved }\end{array}$ & $\begin{array}{l}\text { June } \\
2011\end{array}$ & \begin{tabular}{|c|} 
Not \\
Approved \\
\end{tabular} & NA & NA & No & NA \\
\hline Xtandi & Enzalutamide & $\begin{array}{l}\text { Metastatic castration- } \\
\text { resistant prostate } \\
\text { cancer }\end{array}$ & Oral & $\begin{array}{l}\text { August } \\
2012\end{array}$ & $\begin{array}{l}\text { June } \\
2013\end{array}$ & $\begin{array}{l}\text { June } \\
2013\end{array}$ & $\begin{array}{c}\text { Not } \\
\text { Approved }\end{array}$ & Yes & No & Yes & NA \\
\hline Yervoy & Ipilimumab & Metastatic melanoma & Injectable & $\begin{array}{l}\text { March } \\
2011\end{array}$ & $\begin{array}{l}\text { July } \\
2011\end{array}$ & $\begin{array}{l}\text { March } \\
2012\end{array}$ & $\begin{array}{l}\text { June } \\
2011\end{array}$ & Yes & No & Yes & Yes \\
\hline Zaltrap & Ziv-aflibercept & $\begin{array}{l}\text { Metastatic colorectal } \\
\text { cancer }\end{array}$ & Injectable & $\begin{array}{l}\text { August } \\
2012\end{array}$ & $\begin{array}{c}\text { February } \\
2013\end{array}$ & \begin{tabular}{|c|} 
Not \\
Approved \\
\end{tabular} & $\begin{array}{l}\text { April } \\
2013\end{array}$ & Yes & Yes & NA & No \\
\hline Zelboraf & Vemurafenib & BRAFm+melanoma & Oral & $\begin{array}{l}\text { August } \\
2011\end{array}$ & \begin{tabular}{|c|} 
February \\
2012
\end{tabular} & $\begin{array}{l}\text { March } \\
2012\end{array}$ & $\begin{array}{l}\text { May } \\
2012\end{array}$ & Yes & Yes & Yes & No \\
\hline Zytiga & Abiraterone & Prostate cancer & Oral & $\begin{array}{l}\text { May } \\
2011\end{array}$ & $\begin{array}{c}\text { September } \\
2011\end{array}$ & $\begin{array}{l}\text { July } \\
2011\end{array}$ & $\begin{array}{l}\text { March } \\
2012\end{array}$ & Yes & Yes & Yes & Yes \\
\hline
\end{tabular}

Sources: The FDA and CenterWatch websites were used to identify drugs approved by the FDA for the treatment of any cancer between the dates shown above (http://www.fda.gov/; http://www.centerwatch.com/).

Notes: The date December 31, 2013, was used as the end point for approval decisions, and June 30, 2014, was used as the end point for coverage decisions in non-U.S. countries. NA denotes not applicable because these drugs were not approved in other countries, so they were not covered.

EMA = European Medicines Agency; FDA=U.S. Food and Drug Administration. 
Comparing the Approval and Coverage Decisions of New Oncology Drugs in the United States and Other Selected Countries

\section{FIGURE 1 Comparison of Approval and Coverage Decisions in Other Countries as Percentages of Drugs That} Were Approved in the United States and Covered by Medicare, by Route of Drug Administration

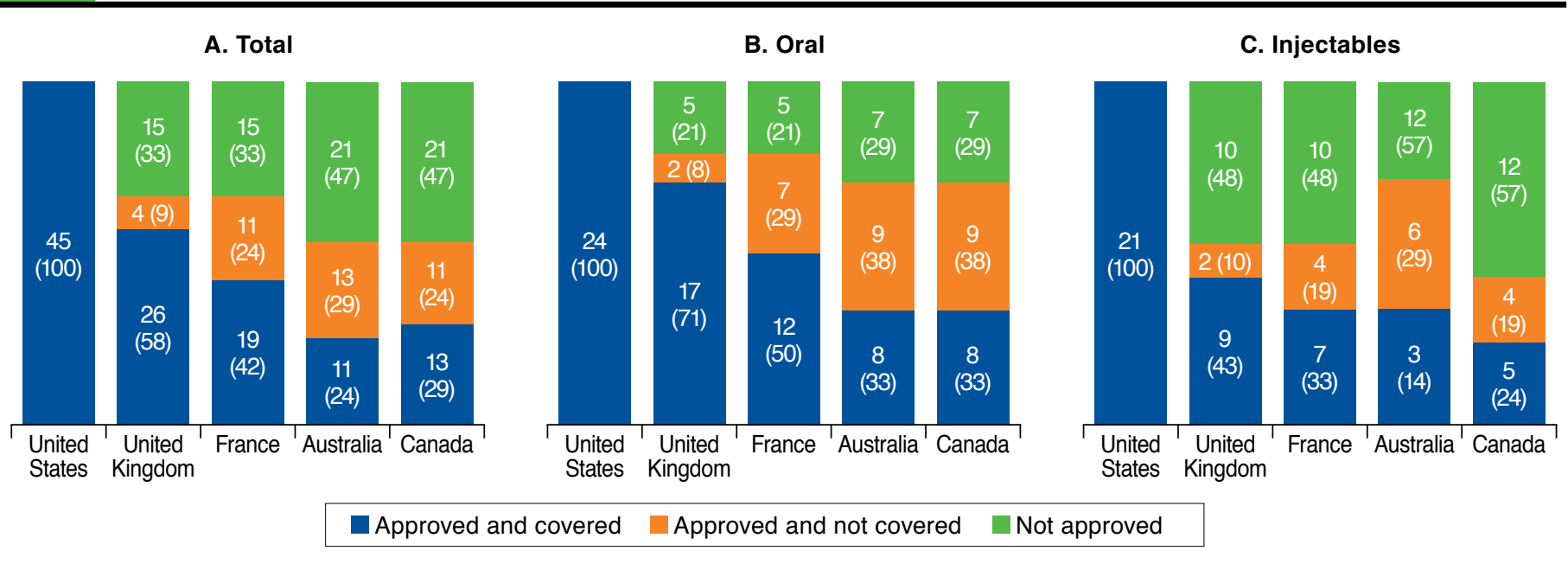

Sources: For the United Kingdom, we reviewed the National Institute for Health and Care Excellence (https://www.nice.org.uk/) and the British National Formulary

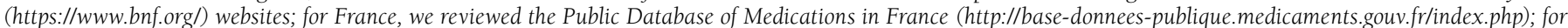

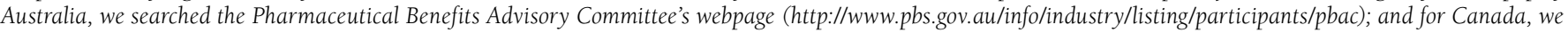

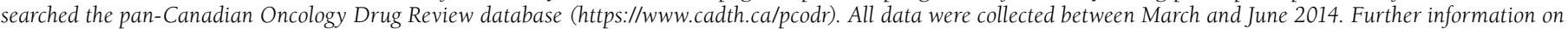
the algorithms used to identify coverage is available upon request.

Note: Results are expressed as $n$ (\%).

approved by the FDA from 2004 through $2008 .^{10}$ In our study, we updated the list of anticancer drugs approved between 2009 and 2013 and found that the proportion of covered medications increased from $42 \%$ as reported by Mason et al. to $58 \%$. Wilson et al. (2011) compared the cancer drug coverage decisions of the United States and Australia for 34 drugs approved by the FDA between 2000 and 2009, and they found that 35\% of the drugs approved by the FDA were approved and covered in Australia. ${ }^{33}$ During our study period, we found that the proportion of covered medications decreased from $35 \%$ to $24 \%$.

Of the 5 countries we reviewed, Australia, Canada, and the United Kingdom used cost-effectiveness analyses explicitly in their coverage decisions. France considered cost implicitly and did not compare health gains against an explicit and rigid cost-effectiveness threshold. Use of cost-effectiveness in coverage decisions was relatively limited in the United States, as compared with the other 4 countries. Medicare has used costeffectiveness analysis to inform its coverage decisions for preventive care services, but it does not and is unlikely that it will use explicit and rigid cost-effectiveness thresholds in coverage decisions for treatments, as in the United Kingdom. ${ }^{34}$ Nevertheless, allowing the flexible use of cost-effectiveness analysis to guide some reimbursement policies may be beneficial, especially for conditions with multiple treatment options and existing rigorous comparative effectiveness evidence. For example, there can be flexibilities in different thresholds for different subgroups, different conditions, how well patients respond to the treatment, and patient life expectancy. ${ }^{35}$ In fact, under the U.S. Affordable Care Act, the payment and integrated delivery models incentivize high-value cancer care, ranging from preventive screening, to value-based treatment, and to palliative care to end-of-life patients. For example, in the Medicare program, accountable care organization providers have incentives to use low-cost and high-value oncology care to manage their patients' health. ${ }^{36}$

\section{Limitations}

There are several limitations in this study. First, the results on the comparative coverage of anticancer drugs in 5 countries are not generalizable to other therapeutic classes. Coverage of anticancer drugs is likely to differ from other therapeutic classes because cancer is often a life-threatening illness. Second, we did not evaluate when manufacturers actually submitted the approval requests to the approval agencies of the different countries. If manufacturers did not submit to the EMA during the study period, we would not have been able to observe those approval dates. However, previous researchers have shown that manufacturers often submitted to the FDA and the EMA around the same time and submitted to Australian and Canadian agencies within 3 months after submission to the FDA. ${ }^{37}$ Third, we only reported whether a drug was covered in a specific country during our study period and did not report 
actual coverage dates because countries do not routinely report these dates. Finally, we did not compare cancer outcomes across countries.

\section{Conclusions}

Of 45 anticancer drug indications approved in the United States between January 1, 2009, and December 31, 2013, 67\% (30) were approved by the EMA, and 53\% (24) were approved in Canada and Australia before December 31, 2013. As of June 30, 2014, in the United States, Medicare covered all 45 drug indications, while the United Kingdom, France, Canada, and Australia covered 58\% (26), 42\% (19), 29\% (13), and 24\% (11) of that number, respectively.

\section{Authors}

YUTING ZHANG, PhD, Department of Health Policy and Management, Graduate School of Public Health, University of Pittsburgh, Pittsburgh, Pennsylvania; HANA CHANTEL HUESER, Department of Biological Sciences, Dietrich School of Arts and Sciences, University of Pittsburgh, Pittsburgh, Pennsylvania; and INMACULADA HERNANDEZ, PharmD, PhD, Department of Pharmacy and Therapeutics, School of Pharmacy, University of Pittsburgh, Pittsburgh, Pennsylvania.

AUTHOR CORRESPONDENCE: Yuting Zhang, PhD, Department of Health Policy and Management, University of Pittsburgh, 130 De Soto St., Crabtree Hall A664, Pittsburgh, PA 15261. Tel.: 412.383.5340; E-mail: ytzhang@pitt.edu.

\section{DISCLOSURES}

No outside funding supported this study, and the authors report no conflicts of interest.

Study concept and design were contributed primarily by Zhang, along with Hernandez and Hueser. All authors participated in data collection, and data interpretation was performed by Zhang and Hernandez, along with Hueser. The manuscript was written and revised by Zhang and Hernandez, along with Hueser.

\section{ACKNOWLEDGMENTS}

The authors thank Andre Lebrun for his excellent research assistance in collecting information on the approval and coverage of oncologics in Australia.

\section{REFERENCES}

1. World Health Organization. Cancer. Fact sheet. Updated February 2015. Available at: http://www.who.int/mediacentre/factsheets/fs297/en/. Accessed January 5, 2017.

2. Khayat D. Innovative cancer therapies: putting costs into context. Cancer. 2012;118(9):2367-71

3. Statista. Top 20 therapeutic classes by global pharmaceutical sales in 2015 (in billion U.S. dollars). Available at: http://www.statista.com/statistics/279916/top-10-therapeutic-classes-by-global-pharmaceutical-sales/. Accessed January 5, 2017.
4. Roberts SA, Allen JD, Sigal EV. Despite criticism of the FDA review process, new cancer drugs reach patients sooner in the United States than in Europe. Health Aff (Millwood). 2011;30(7):1375-81.

5. The Commonwealth Fund. Mirror, mirror on the wall, 2014 update: how the U.S. health care system compares internationally. June 16, 2014. Available at: http://www.commonwealthfund.org/publications/fundreports/2014/jun/mirror-mirror. Accessed January 5, 2017.

6. World Health Organization. The World Health Report 2000. Health Systems: Improving Performance. Geneva, Switzerland: World Health Organization; 2000. Available at: http://www.who.int/whr/2000/en/whr00_en.pdf. Accessed January 5, 2017.

7. U.S. Food and Drug Administration. How drugs are developed and approved. Updated August 18, 2015. Available at: http://www.fda.gov/ Drugs/Development ApprovalProcess/HowDrugsareDevelopedandApproved/ Accessed January 5, 2017.

8. Bach PB. Limits on Medicare's ability to control rising spending on cancer drugs. N Engl J Med. 2009;360(6):626-33.

9. Patients Equal Access Coalition. Oral chemotherapy access legislative map. 2016. Available at: http://peac.myeloma.org/oral-chemo-access-map/. Accessed January 5, 2017.

10. Mason A, Drummond M, Ramsey S, Campbell J, Raisch D. Comparison of anticancer drug coverage decisions in the United States and United Kingdom: does the evidence support the rhetoric? J Clin Oncol. 2010;28(20):3234-38

11. Raftery J. Review of NICE's recommendations, 1999-2005. Br Med J. 2006;332(7552):1266.

12. Jonsson B. Technology assessment for new oncology drugs. Clin Cancer Res. 2013;19(1):6-11.

13. Department of Health. The pharmaceutical price regulation scheme 2014. December 2013. Available at: https://www.gov.uk/government/ uploads/system/uploads/attachment_data/file/282523/Pharmaceutical_ Price_Regulation.pdf. Accessed January 5, 2017.

14. Williamson S. Patient access schemes for high-cost cancer medicines. Lancet Oncol. 2010;11(2):111-12.

15. National Institute for Health and Care Excellence. List of technologies with approved Patient Access Schemes. 2014. Available at: https://www.nice. org.uk/about/what-we-do/patient-access-schemes-liaison-unit/list-of-technologies-with-approved-patient-access-schemes. Accessed January 5, 2017.

16. L'agence nationale de sécurité du médicament et des produits de santé. Conseil Scientifique. 2015. Available at: http://ansm.sante.fr/L-ANSM2/ Gouvernance/Conseil-scientifique/(offset)/2. Accessed January 5, 2017.

17. Haute Autorité de Santé. Reglement Interieure de la Commission de la Transparence (Rules of the Interior Committee). Updated April 6, 2016. Available at: http://www.has-sante.fr/portail/upload/docs/application/ pdf/2013-01/ri_ct_version_07112012_vf_f.pdf. Accessed January 15, 2017.

18. Santé-Médecine. Liste des affections de longue durée (ALD). 2014. Available at: http://sante-medecine.commentcamarche.net/faq/477-liste-desaffections-de-longue-duree-ald. Accessed January 5, 2017.

19. Therapeutic Goods Administration. TGA basics. 2014. Available at: http://www.tga.gov.au/about/tga.htm\#.UzHVmj45NJU. Accessed January 5, 2017.

20. Therapeutic Goods Administration. About the work of the TGA - a risk management approach. August 15, 2011. Available at: http://www.tga.gov. au/about/tga-risk-management-approach.htm\#.UzHWez45NJU. Accessed January 5, 2017.

21. Simon SR, Rodriguez HP, Majumdar SR, et al. Economic analysis of a randomized trial of academic detailing interventions to improve use of antihypertensive medications. J Clin Hypertens. 2007;9(1):15-20. 
22. IMS Institute for Healthcare Informatics. Impact of cost-per-QALY reimbursement criteria on access to cancer drugs. December 2014. Available at: https://www.imshealth.com/files/web/IMSH\%20Institute/Healthcare\%20 Briefs/IHII_CPQ_Impact_on_Access_to_Cancer_Drugs.pdf. Accessed January $5,2017$.

23. Morgan S, Mintzes B, Barer M. The economics of direct-to-consumer advertising of prescription-only drugs: prescribed to improve consumer welfare? J Health Sev Res Policy. 2003;8(4):237-44.

24. Canadian Agency for Drugs and Technologies in Health. CADTH Common Drug Review. 2014. Available at: https://www.cadth.ca/aboutcadth/what-we-do/products-services/cdr. Accessed January 15, 2017.

25. Canadian Agency for Drugs and Technologies in Health. CADTH panCanadian Oncology Drug Review (pCODR). 2011. Available at: https://www. cadth.ca/pcodr. Accessed January 15, 2017.

26. Downing NS, Aminawung JA, Shah ND, Braunstein JB, Krumholz HM, Ross JS. Regulatory review of novel therapeutics - comparison of three regulatory agencies. New Engl J Med. 2012;366(24):2284-93.

27. Roberts SA, Allen JD, Sigal EV. Despite criticism of the FDA review process, new cancer drugs reach patients sooner in the United States than in Europe. Health Aff (Millwood). 2011;30(7):1375-81.

28. British National Formulary. Website. 2014. Available at: https://www. bnf.org/. Accessed January 15, 2017.

29. Ministère des Affaires sociales et de la Santé. Base de données publique des médicaments. 2014. Available at: http://base-donnees-publique.medicaments.gouv.fr/index.php. Accessed January 5, 2017.
30. Agence Technique de l'Information sur l'Hospitalisation (Technical Agency for Hospital Information). Liste des unités communes de dispensation prises en charge en sus. 2015. Available at: http://www.atih.sante. fr/liste-des-unites-communes-de-dispensation-prises-en-charge-en-sus. Accessed January 5, 2017.

31. Australian Department of Health. Pharmaceutical Benefits Scheme. 2014. Available at: http://www.pbs.gov.au/pbs/home. Accessed January 5, 2017.

32. Canadian Agency for Drugs and Technologies in Health. CADTH panCanadian Oncology Drug Review. 2014. Available at: https://www.cadth.ca/ pcodr. Accessed January 5, 2017.

33. Wilson A, Cohen J. Patient access to new cancer drugs in the United States and Australia. Value Health. 2011;14(6):944-52.

34. Neumann PJ, Rosen AB, Weinstein MC. Medicare and cost-effectiveness analysis. N Engl J Med. 2005;353(14):1516-22.

35. Neumann PJ, Cohen JT, Weinstein MC. Updating cost-effectivenessthe curious resilience of the \$50,000-per-QALY threshold. N Engl J Med. 2014;371(9):796-97.

36. Mehta AJ, Macklis RM. Overview of accountable care organizations for oncology specialists. J Oncol Pract. 2013;9(4):216-21.

37. Centre for Innovation in Regulatory Science. The impact of the changing regulatory environment on the approval of new medicines across six major authorities 2004-2013. December 16, 2014. Available at: http://cirsci. org/sites/default/files/R\&D\%20Briefing\%2055\%2016122014.pdf. Accessed January 5, 2017. 